\title{
NA49 and NA61/SHINE experiments: results and plans
}

\author{
Tatjana Šuša for the NA49 and NA61 Collaborations \\ Rudjer Boskovic Institute, Bijenička cesta 54, 10000 Zagreb, Croatia
}

DOI: http://dx.doi.org/10.5689/UA-PROC-2010-09/43

\begin{abstract}
Results of the search for the onset of deconfinement and the critical point performed by the NA49 experiment at the CERN SPS facility are reviewed, and the status and plans of its successor NA61/SHINE experiment are outlined.
\end{abstract}

\section{Introduction}

Quantum chromodynamics predicts that at sufficiently high energy density $\left(\epsilon \approx 1 \mathrm{GeV} / \mathrm{fm}^{3}\right)$ strongly interacting matter will undergo a phase transition from hadronic matter to a state characterized by partonic degrees of freedom, the quark-gluon plasma (QGP) [1]. The NA49 experiment [2] has studied central $\mathrm{Pb}+\mathrm{Pb}$ collisions at different energies $(20,30,40,80$ and 158 $\mathrm{A} \mathrm{GeV}$ ) with the aim to search for the onset of deconfinement i.e. the lowest energy sufficient to create the predicted partonic state. Final results of the energy scan program will be reviewed.

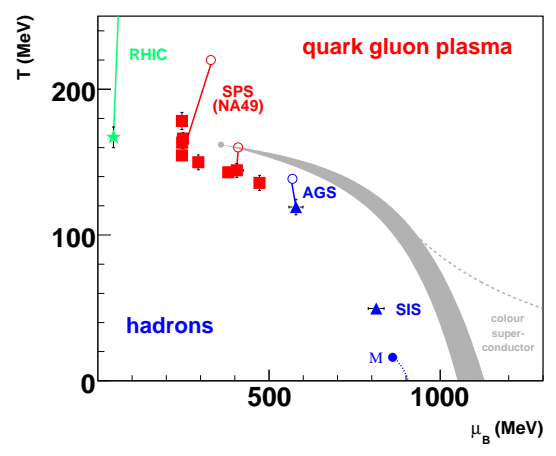

Figure 1: Phase diagram of strongly interacting matter. The line indicates first order phase transition ending in the critical point. Symbols denote chemical freeze-out parameters extracted from hadron gas model fits [5].

ISMD2010
Theoretical studies of the QCD phase diagram $[3,4]$ predict a first order transition at large baryochemical potential, which changes to a crossover at small $\mu_{B}$. This implies the existence of a critical point at the end of the first order transition line (Figure 1). The recent lattice calculation locate the critical point in the SPS energy range [6]. If freeze-out of a strongly interacting system occurs close to the critical point, large nonstatistical fluctuations are expected. The results of the search for predicted signatures of critical point in central $\mathrm{Pb}+\mathrm{Pb}$ collisions at different energies and in $\mathrm{p}+\mathrm{p}$ and semicentral $\mathrm{C}+\mathrm{C}$ and $\mathrm{Si}+\mathrm{Si}$ collisions at top SPS energy will be presented.

The NA49 successor, NA61/SHINE experiment will continue the search for the critical point and explore in detail the onset of deconfinement. The current status and plans of the experiment will be summarized. 


\section{Onset of deconfinement}

The NA49 energy scan program was motivated by the predictions of the Statistical Model of the Early Stage (SMES) [7]. Within the model the transition to the deconfined state is predicted to occur between 30 and $60 \mathrm{AGeV}$ and manifest itself via the anomalies in the energy dependence of various hadron production properties.
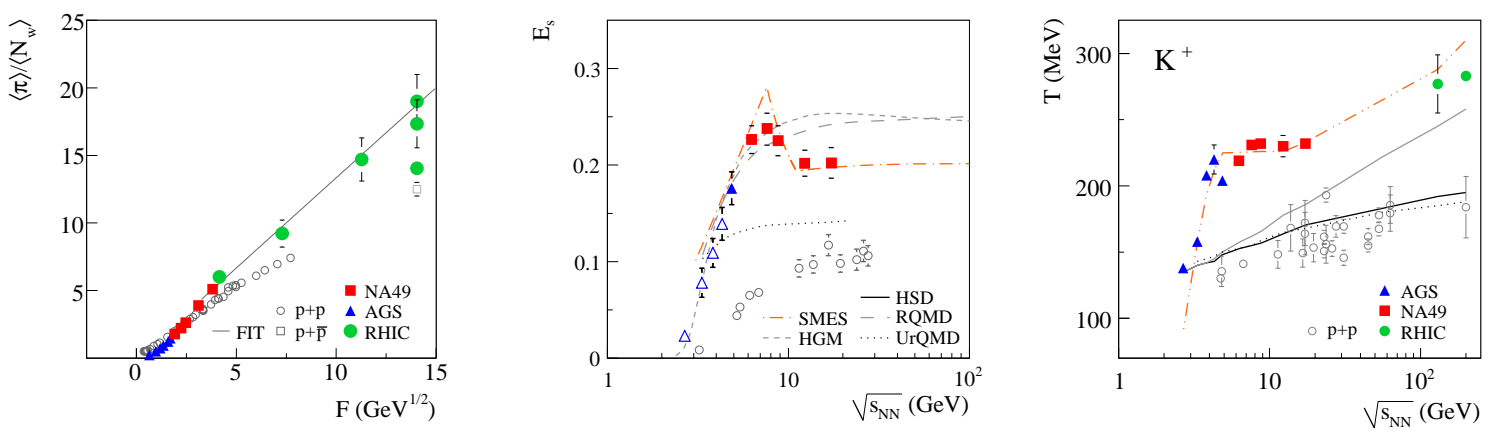

Figure 2: Total pion yield per wounded nucleon as a function of Fermi variable $\mathrm{F} \approx s_{N N}^{1 / 4}$ (left). Strangeness to pion ratio $E_{S}=(\langle K\rangle+\langle\Lambda\rangle) /\langle\pi\rangle$, as a function of collision energy (center). Inverse slope parameter $T$ of of $K^{+}$mesons versus collision energy (right).

The key results $[8,9]$ of the energy scan program are summarized in Figure 2. The total pion yield normalized to the number of wounded nucleons steepens in the SPS energy range, the strangeness to pion ratio, $\left.E_{S}=(\langle\Lambda\rangle+\langle K+\bar{K}\rangle) / \pi\right)$ exhibits a sharp pronounced maximum around $30 \mathrm{AGeV}$, while the slope of the $\mathrm{K}^{+}$transverse momentum distributions shows an approximately constant value after a rapid rise at lower energies. These features are neither observed in elementary $\mathrm{p}+\mathrm{p}(\bar{p})$ interactions (open symbols in Figure 2) nor are reproduced in hadronic transport models or statistical models of hadron production. The data are consistent with the expected signals of the onset of a phase transition at low SPS energies.

\section{Search for the critical point}

Event-by-event fluctuations (in particular of particle multiplicity and mean transverse momentum) are suggested as the observables sensitive to the presence of critical point. The size of the fluctuation signal is predicted to change non-monotonically with energy or size of the colliding system and to reach maximum when the freeze-out occurs in the vicinity of the critical point. The NA49 experiment has searched for the critical point by studying energy and system size dependence of particle multiplicity $[10,11]$, mean transverse momentum $\left\langle p_{T}\right\rangle[12]$ and azimuthal angle [13] fluctuations.

Particle multiplicity fluctuations are characterized by the scaled variance $\omega$ of the multiplicity distribution [10], while for the characterization of $\left\langle p_{T}\right\rangle$ fluctuations the $\Phi_{p_{T}}$ measure [12] is used. System size and energy dependence of $\omega$ and $\Phi_{p_{T}}$ are shown in Figure 3. Results for different energies and nuclear masses are plotted as a function of baryochemical potential $\mu_{B}$ and temperature $T_{\text {chem }}$ extracted from the hadron gas model fits to particle yields [5]. The 

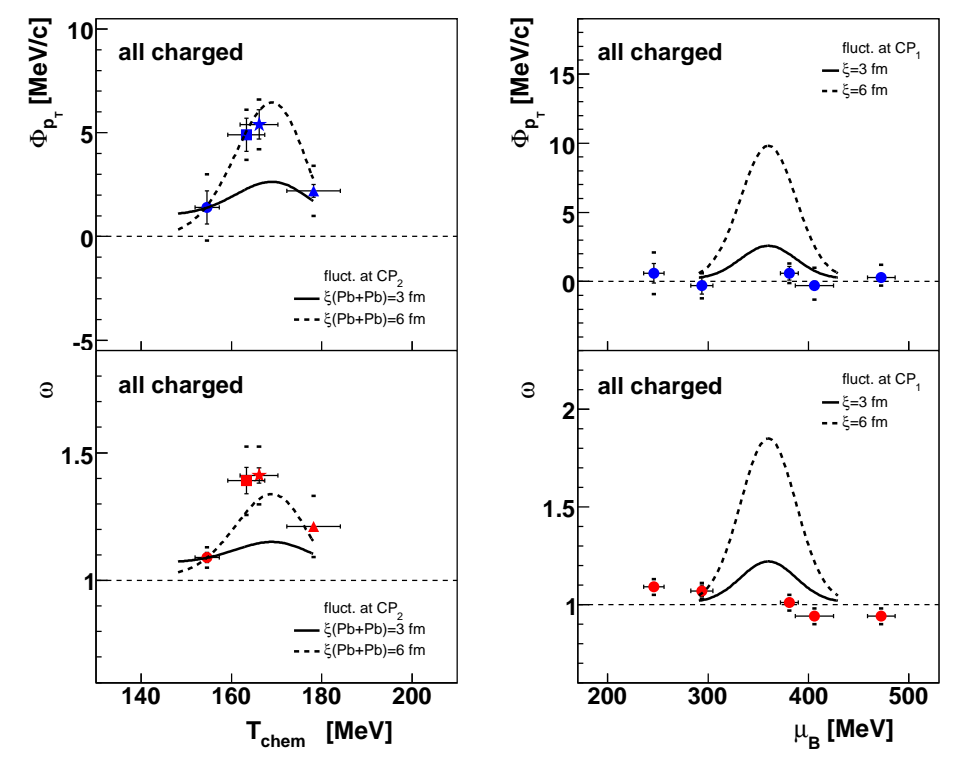

Figure 3: System size $\left(T_{\text {chem }}\right)$ and energy $\left(\mu_{B}\right)$ dependence of $\omega$ and $\Phi_{p_{T}}$. Dashed and solid lines indicate estimated effect of critical point for two values of correlation length.

experimental results are compared to the estimated effect of the critical point for two values of correlation length, $\xi=6 \mathrm{fm}$ and $\xi=3 \mathrm{fm}$. Two locations of the critical point are considered: $\mathrm{CP} 1\left(\mathrm{~T}=147 \mathrm{MeV}, \mu_{B}=360 \mathrm{MeV}\right)$ and $\mathrm{CP} 2\left(\mathrm{~T}=178 \mathrm{MeV}, \mu_{B}=250 \mathrm{MeV}\right)$, for details see [14]. No indications of the CP1 point is observed in the energy dependence of multiplicity and $\left\langle p_{T}\right\rangle$ fluctuations. On the other hand, the system size dependence of both multiplicity and $\left\langle p_{T}\right\rangle$ fluctuations, is consistent with CP2 predictions.

Figure 4 shows the energy and system size dependence of the third moment $\Phi_{P_{T}}^{(3)}[15]$ of the $\left\langle p_{T}\right\rangle$ fluctuations, which is expected to be more sensitive to the presence of nonstatistical fluctuations. Results are plotted for all, and for positive and negative charged particles separately. Although, there is no quantitative estimations for the effect of critical point and the systematic errors are still large due to the missing two track resolution correction, no indication of the critical point is observed.

Event-by-event fluctuations of azimuthal angle are sensitive to the plasma instabilities [16], fluctuations of elliptic flow [17] as well as the presence of the critical point. NA49 results on energy and system size dependence of azimuthal angle fluctuations are plotted in Figure 5. No significant energy dependence is observed whereas weak indication of the maximum is visible in the nuclear size dependence. 

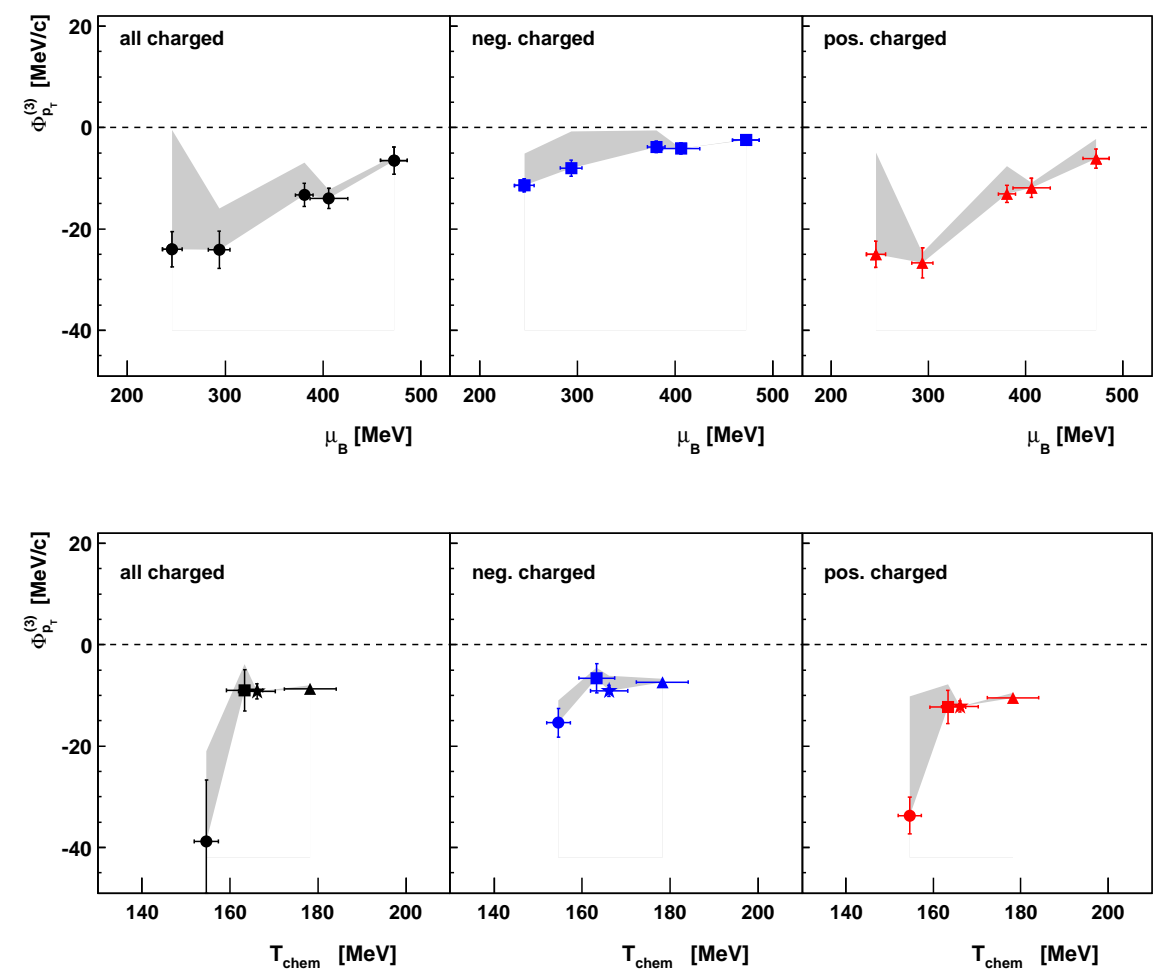

Figure 4: Third moment $\Phi_{P_{T}}^{(3)}$ of $\left\langle p_{T}\right\rangle$ fluctuations versus energy $\left(\mu_{B}\right)$ and system size $\left(T_{\text {chem }}\right)$ for all charged and for positive and negative charged particles separately.

\section{NA61/SHINE experiment}

The NA49 observation of the onset of deconfinement in central $\mathrm{Pb}+\mathrm{Pb}$ collisions at the low SPS energies and the predictions of lattice QCD calculations which locate the critical point in the SPS energy range have motivated the continuation of the heavy ion program at the CERN SPS which will be performed by the NA61/SHINE experiment. The NA61/SHINE experiment aims to search systematically for the critical point of strongly interacting matter and study in detail the properties of the onset of deconfinement. These physics goals will be pursued by performing a two-dimensional scan in $\left(T-\mu_{B}\right)$ of the phase diagram by varying collision energy (13A$158 \mathrm{~A} \mathrm{GeV})$ and size of the colliding systems ( $\mathrm{p}+\mathrm{p}, \mathrm{p}+\mathrm{Pb}, \mathrm{B}+\mathrm{C}, \mathrm{Ar}+\mathrm{Ca}, \mathrm{Xe}+\mathrm{La})$. In addition to these main objectives, the experiment will establish the energy dependence of the nuclear modification factor and obtain precision data on hadron spectra in hadron-nucleus collisions for the T2K neutrino experiment, and for the Pierre Auger Observatory and KASCADE cosmic-ray experiments.

In order to accomplish physics goals of the new experimental program, several upgrades of the detector apparatus inherited from the NA49 are performed and foreseen. In 2007, a forward Time-of-Flight was constructed in order to extend the acceptance for particles with 

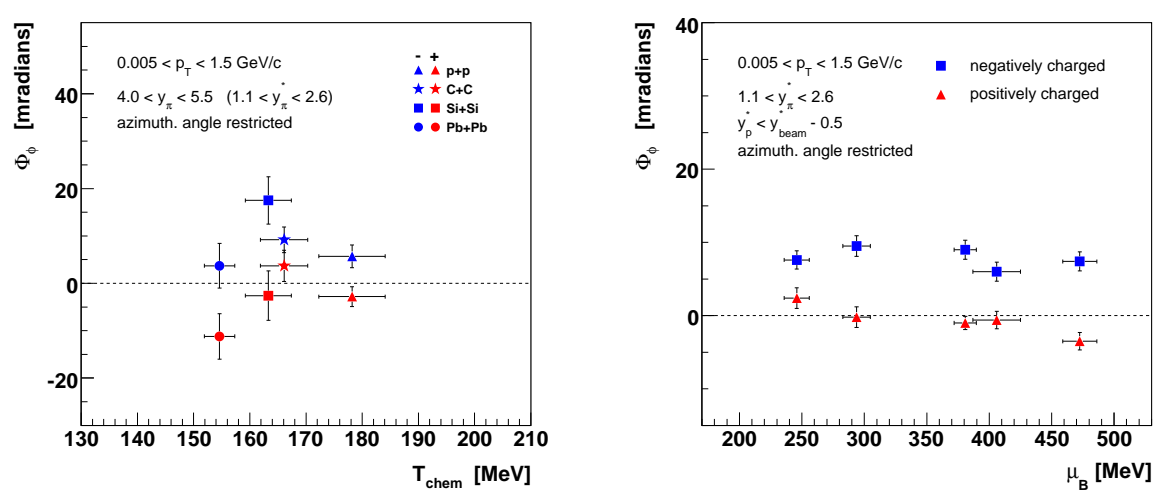

Figure 5: $\Phi_{\phi}$ measure of azimutahal angle fluctuations as a functon of system size $\left(T_{\text {chem }}\right)$ and energy $\left(\mu_{B}\right)$.

p $<3 \mathrm{GeV} / \mathrm{c}$; in 2008, the DAQ system and the TPC readout electronics were replaced to achieve an increase in the data taking rate by a factor of 10. In 2011, the NA49 Forward Calorimeter will be replaced by the Projectile Spectator Detector which will provide a single nucleon energy resolution essential for the fluctuation studies. For 2011, also the installation of the Helium beam pipe around the beam inside the sensitive TPC volume is foreseen as well as the conversion of the $\mathrm{H} 2$ beam line into a fragment separator. The latter change is necessary in order to run with low mass ions (boron) in parallel with the LHC Pb program.

The NA61/SHINE data taking schedule is summarized in Table 1. The data taking started in 2007 with the pilot $p+C$ run at $31 \mathrm{GeV} / \mathrm{c}$ beam energy. The preliminary pion spectra from the pilot run were shown at several conferences $[18,19,20,21,22]$ and will be published soon. $\mathrm{p}+\mathrm{C}$ and $\pi+\mathrm{C}$ data needed for the $\mathrm{T} 2 \mathrm{~K}$ and cosmic ray experiments were collected in 2009 and 2010. The first part of the system size and energy scan program, namely $\mathrm{p}+\mathrm{p}$ collisions at 13 , 20, 30, 40, 80 and $158 \mathrm{GeV} / \mathrm{c}$ beam energy were/will be taken in 2009, 2010 and 2011. B+C, $\mathrm{Ar}+\mathrm{Ca}$ and $\mathrm{Xe}+\mathrm{La}$ runs are foreseen for 2011, 2013 and 2014, respectively. p+Pb interactions will be recorded in 2013 and 2014 .

\section{Conclusion}

The NA49 experiment has obtained evidence for the onset of deconfinement at $30 \mathrm{~A} \mathrm{GeV}$ beam energy by analysing energy dependence of hadron production properties in central $\mathrm{Pb}+\mathrm{Pb}$ collisions. Present studies of the system size and energy dependence of particle multiplicity, mean transverse momentum and azimuthal angle fluctuations do not allow to draw the firm conclusion about the existence and location of the critical point.

The NA61/SHINE experiment will continue the search for the critical point and study in detail the onset of deconfinement by performing a two dimensional scan of the QCD phase diagram. This program was stared in 2009 with the measurements of $p+p$ interactions. Low mass ion $(\mathrm{B}+\mathrm{C})$ runs are foreseen for 2011, while $\mathrm{Ar}+\mathrm{Ca}$ and $\mathrm{Xe}+\mathrm{La}$ interactions are planned for 2013 and 2014, respectively. 


\begin{tabular}{ccccc}
\hline Beam+Target & Energy $(\mathrm{AGeV})$ & Year & Physics & Status \\
\hline $\mathrm{p}+\mathrm{C}$ & 30 & 2007 & T2K, CR & performed \\
$\pi^{-}+\mathrm{C}$ & 158,350 & 2009 & $\mathrm{CR}$ & performed \\
$\mathrm{p}+\mathrm{C}$ & 30 & $2009 / 2010$ & T2K, CR & performed \\
$\mathrm{p}+\mathrm{p}$ & $13,20,30,40,80,158$ & $2009 / 10 / 11$ & $\mathrm{CP}, \mathrm{OD}$, high $p_{T}$ & in progress \\
$\mathrm{B}+\mathrm{C}$ & $13,20,30,40,80,158$ & 2011 & $\mathrm{CP}, \mathrm{OD}$ & to be discussed \\
$\mathrm{Ar}+\mathrm{Ca}$ & $13,20,30,40,80,158$ & 2013 & $\mathrm{CP}, \mathrm{OD}$ & to be discussed \\
$\mathrm{p}+\mathrm{Pb}$ & 158 & $2013 / 2014$ & $\mathrm{CP}, \mathrm{OD}$, high $p_{T}$ & to be discussed \\
$\mathrm{X}+\mathrm{La}$ & $13,20,30,40,80,158$ & 2014 & $\mathrm{CP}, \mathrm{OD}$ & to be discussed \\
\hline
\end{tabular}

Table 1: NA61/SHINE data taking schedule. The physics goals of different data taking periods are denoted by the following abbreviations: CP - search for the critical point, OD - study of the onset of deconfinement, high $p_{T}$ - reference spectra for nuclear modification factors, CR precision data for cosmic ray experiments, $\mathrm{T} 2 \mathrm{~K}$ - precision data for $\mathrm{T} 2 \mathrm{~K}$ experiment.

\section{Acknowledgments}

I would like to thank to the ISMD2010 organisers for the invitation and the opportunity to show results from NA49 and status of NA61/SHINE experiment.

\section{References}

[1] E. V. Shuryak, Phys. Rep.61 (1980) 71.

[2] S. Afanasiev et al., Nucl. Instrum. Meth. A430 (1999) 210.

[3] J. I. Kapusta, A. Mekjian, Phys. Rev. D 33 (1986) 1304.

[4] M. A. Stephanov, K. Rajagopal, E. V. Shuryak, Phys. Rev. Lett. 81 (1998) 4816, M. Stephanov, Prog. Theor. Phys. Suppl. 153 (2004) 139.

[5] F. Becattini, J. Manninen, M. Gazdzicki, Phys. Rev. C 73 (2006) 044905 [arXiv:hep-ph/0511092].

[6] Z. Fodor, S. Katz, J. High Energy Phys. 0404 (2004) 50.

[7] M. Gadzicki, M. Gorenstein, Acta Phys. Polon. B 30 (1999) 2705.

[8] S. V. Afanasiev et al., Phys. Rev. C 66 (2002) 054902.

[9] C. Alt et al., Phys. Rev. C 77 (2008) 024903.

[10] C. Alt et al., Phys. Rev. C 78, (2008) 034914.

[11] C. Alt et al., Phys. Rev. C 75, (2007) 064904.

[12] C. Anticic et al., Phys. Rev. C 79, (2009) 044904.

[13] T. Cetner et al., arXiv:1008.3412 [nucl-ex] (2010).

[14] K. Grebieszkow et al., arXiv:0907.4101v2 [nucl-ex] (2009) .

[15] S. Mrówczyński, Phys.Lett.B 465 (1999)8

[16] S. Mrówczyński, Phys. Lett. B 314 (1993) 118

[17] S. Mrówczyński, E. Shuryak, Acta Phys. Polon. B 314 (2003) 4241.

[18] M. Z. Posiadala, Acta Phys. Polon. B 41 (2010) 1585.

[19] C. Strabel, arXiv:1006.0767 [hep-ex].

[20] N. Abgrall, arXiv:1005.3692 [hep-ex].

[21] S. Murphy, arXiv:1005.3689 [hep-ex].

[22] T. J. Palczewski, PoS EPS-HEP2009 (2009) 412. 